\title{
LOCAL AND GLOBAL REGULARITY OF WEAK SOLUTIONS OF ELLIPTIC EQUATIONS WITH SUPERQUADRATIC HAMILTONIAN
}

\author{
ANDREA DALL'AGLIO AND ALESSIO PORRETTA
}

\begin{abstract}
In this paper, we study the regularity of weak solutions and subsolutions of second order elliptic equations having a gradient-dependent term with superquadratic growth. We show that, under appropriate integrability conditions on the data, all weak subsolutions in a bounded and regular open set $\Omega$ are Hölder-continuous up to the boundary of $\Omega$. Some local and global summability results are also presented. The main feature of this kind of problem is that the gradient term, not the principal part of the operator, is responsible for the regularity.
\end{abstract}

\section{INTRODUCTION AND MAIN RESULTS}

Recently, several papers have investigated the regularity of solutions of second order (possibly degenerate) equations containing first order terms with superquadratic growth in the gradient. Firstly, motivated by stochastic control problems, in [8] the authors considered fully nonlinear equations whose simplest example is the viscous Hamilton-Jacobi equation

$$
-\operatorname{tr}\left(A(x) D^{2} u\right)+\lambda u+|D u|^{p}=f(x), \quad x \in \Omega,
$$

in an open bounded set $\Omega \subset \mathbb{R}^{N}, N \geq 2$, where $A$ is a continuous nonnegative $N \times N$ symmetric matrix, $f(x)$ is continuous and $\lambda \geq 0$. The main result proved in [8] states that, when $p>2$, any bounded upper semi-continuous viscosity subsolution of (1.1) is Hölder continuous in $\bar{\Omega}$ (under some regularity of $\partial \Omega$ ) of exponent $\alpha=\frac{p-2}{p-1}$, with estimates depending only on the $L^{\infty}$-norm of $A(x)$ and $f(x)$.

This result shows two striking effects of the superquadratic growth of the Hamiltonian; one is that the Hölder regularity holds for merely subsolutions, which is unusual for second order problems. Another one is that the regularity, and the corresponding estimate, carry over up to the boundary, which explains why the Dirichlet problem can be overdetermined for this kind of operator. This is a major difference with the case that first order terms have the so-called natural growth, meaning that they grow at most quadratically with respect to the gradient (for this case see [3], [10] and the references therein). Otherwise, some peculiarities of the superquadratic case had been pointed out in the pioneering works [13, [11, at least concerning properties of solutions.

Received by the editors May 7, 2012 and, in revised form, October 4, 2012.

2010 Mathematics Subject Classification. Primary 35B65, 35J60; Secondary 35R45, 35R05.

Key words and phrases. Elliptic equations, superquadratic growth, Hölder continuity of solutions, local estimates, boundedness of solutions. 
The regularity result of [8], mentioned before, was revisited in [2], where an interpretation was given in terms of state-constraint problems together with several possible applications. At the same time, the regularity of solutions for the corresponding evolution equations was investigated in [5], 4] and next in [7, 6], where Hölder regularity and estimates of (viscosity) solutions were proved for several types of second order, possibly degenerate, time-dependent operators (both local and nonlocal) with the common feature of a superquadratic coercive gradient dependent lower order term.

The goal of our paper is to prove similar estimates and regularity results for stationary distributional solutions of second order, possibly degenerate, operators in divergence form. Since all previous works have concerned the framework of viscosity solutions, our results complement those cited above and show, once more, the generality of the Hölder regularity induced by the superquadratic term. Let us stress that distributional solutions in this context are not unique (see the discussion in Remark 3.2), therefore the regularity proved in this class has a stronger flavour. Indeed, we show that similar results to those proved in [8] hold even in the weak context of distributional solutions, for the divergence form structure, and if $f$ belongs to a (larger) class of Lebesgue spaces. In order to be more precise, here is our main result.

Theorem 1.1. Let $\Omega$ be an open bounded and connected subset of $R^{N}$ having Lipschitz boundary and satisfying the uniform interior sphere condition. Assume $a(x, s, \xi)$ is a Carathéodory function satisfying, for some $\beta>0$,

$$
|a(x, s, \xi)| \leq \beta(1+|\xi|) \quad \forall(s, \xi) \in \mathbb{R} \times \mathbb{R}^{N} \text {, a.e. } x \in \Omega .
$$

Let $p>2, \lambda \geq 0$ and let $f$ belong to $L^{q}(\Omega)$ for some $q>\frac{N}{p}$. Let $u$ be a function in $W_{\text {loc }}^{1, p}(\Omega)$ such that $\lambda u^{-} \in L^{q}(\Omega)$, which satisfies, in the sense of distributions, the inequality

$$
\lambda u+|\nabla u|^{p} \leq \operatorname{div}(a(x, u, \nabla u))+f(x) \quad \text { in } \Omega .
$$

Then $u$ is Hölder continuous in $\bar{\Omega}$ (i.e., up to the boundary) and satisfies

$$
|u(x)-u(y)| \leq K|x-y|^{\alpha}, \quad \forall x, y \in \bar{\Omega},
$$

where $\alpha=\min \left(1-\frac{N}{p q}, 1-\frac{1}{p-1}\right)$ and $K$ depends on $p, q, N, \beta, \Omega,\|f\|_{L^{q}(\Omega)}$ and $\left\|\lambda u^{-}\right\|_{q}$.

Theorem 1.1] is the natural extension of the main result proved in [8, Thm 1.1]. We recover all the features mentioned before: the operator can be degenerate or not, since the estimate only depends on the $L^{\infty}$-bound of the field $a$, moreover, the estimate holds up to $\partial \Omega$ and, in particular, it is a universal estimate for positive solutions. Note also that the Hölder exponent $\alpha$ decreases according to $q$ if $f \in$ $L^{q}(\Omega)$ with $q<\frac{N(p-1)}{p}$, embedding the $\frac{p-2}{p-1}$-Hölder regularity previously known into a more general scale. Let us mention that the possibility to obtain Hölder estimates with unbounded data $f$ had not been considered in the previous works except for the recent paper [6] for the solutions of evolution problems.

The proof of our result is completely different from the one given in [8], obviously due to the different framework of distributional solutions rather than viscosity solutions. This gives an independent interest to our proof; indeed, the integral approach 
induced by the distributional formulation suggests a different, yet natural, interpretation of the Hölder regularity as an immediate consequence of a local Morrey-type inequality.

Theorem 1.1 is not the only result that we prove. Indeed, we will further prove several local and global different estimates, including the case where $f \in L_{\text {loc }}^{q}(\Omega)$ with $q<\frac{N}{p}$. In order to better clarify the local and global ingredients, the two aspects should be first considered separately, which is the way we have planned our presentation. However, it is important to stress that, for positive solutions, the local bounds extend to global ones without any information on the boundary values. In this respect, to mention a significant consequence of our estimates, we complement Theorem 1.1 with the following.

Theorem 1.2. Let $\Omega$ satisfy the same assumptions as in Theorem 1.1. Assume (1.2), let $p>2, \lambda>0$ and let $f$ belong to $L^{q}(\Omega)$ for some $q>\frac{N}{p}$. Let $u \in W_{\text {loc }}^{1, p}(\Omega)$ be a subsolution of (1.3) in the sense of distributions. Assume in addition that one of the two following conditions holds:

(i) $a(x, s, \xi) \cdot \xi \geq 0$ for every $(s, \xi) \in \mathbb{R} \times \mathbb{R}^{N}$, a.e. $x \in \Omega$.

(ii) $u \geq 0$ in $\Omega$.

Then $u^{+} \in L^{\infty}(\Omega)$ and

$$
\left\|u^{+}\right\|_{L^{\infty}(\Omega)} \leq M
$$

where $M=M\left(\beta, q, p, N, \lambda^{-1}, \Omega,\|f\|_{L^{q}(\Omega)}\right)$.

The global bound on $u^{+}$given by the previous result extends a similar one proved in 11 in the case of the Laplace operator, in connection with the corresponding state constraint problem (see also [2]). On the other hand, the negative part of solutions can be estimated globally only if one controls the boundary data; we restrict ourselves to consider zero boundary data in that case. Such global estimates for the Dirichlet problem are the object of Section 5 .

Let us note that, in order to keep the exposition simple, we have restricted our attention to the case where the second order operator has linear growth (that is, inequality (1.2) holds). However, all the results contained in this article could be extended with little effort to the case where the operator has growth $m-1$, with $m>1$, that is, inequality (1.2) is replaced by

$$
|a(x, s, \xi)| \leq \beta\left(1+|\xi|^{m-1}\right) \quad \forall(s, \xi) \in \mathbb{R} \times \mathbb{R}^{N} \text {, a.e. } x \in \Omega,
$$

provided the exponent $p$ in the gradient term satisfies $p>m$. For instance, one could consider the following differential inequality involving the $m$-laplacian:

$$
\lambda u+|\nabla u|^{p} \leq \operatorname{div}\left(|\nabla u|^{m-2} \nabla u\right)+f(x) \quad \text { in } \Omega,
$$

with $p>m$.

Indeed, the idea that lies behind the proofs is that, from the point of view of regularity, if $p>m$, the second order operator can be "neglected", so the differential inequality (1.4) behaves like its first order counterpart,

$$
\lambda u+|\nabla u|^{p} \leq f(x),
$$

for which regularity follows as a straightforward consequence of Sobolev's inequalities. 


\section{NotATION}

Let $\Omega$ be a bounded open set in $\mathbb{R}^{N}, N \geq 1$. We will consider a differential inequality of the form

$$
-\operatorname{div}(a(x, u, \nabla u))+\lambda u+|\nabla u|^{p} \leq f(x) \quad \text { in } \Omega,
$$

where $a(x, s, \xi): \Omega \times \mathbb{R} \times \mathbb{R}^{N}$ is a Carathéodory function (i.e., measurable in the first variable and continuous in the last two variables) such that

$$
|a(x, s, \xi)| \leq \beta(1+|\xi|), \quad \beta>0,
$$

for every $(s, \xi) \in \mathbb{R} \times \mathbb{R}^{N}$, for a.e. $x \in \Omega$. We also assume in (2.1) that $p>2, \lambda \geq 0$ (although in the last section we will also consider the case $\lambda<0$ ), and $f(x)$ is a measurable function belonging to $L_{\mathrm{loc}}^{q}(\Omega)$, for some $q \geq 1$.

Definition 2.1. We will say that $u \in W_{\text {loc }}^{1, p}(\Omega)$ is a subsolution of (2.1) in the sense of distributions if

$$
\int_{\Omega} a(x, u, \nabla u) \cdot \nabla \varphi d x+\lambda \int_{\Omega} u \varphi d x+\int_{\Omega}|\nabla u|^{p} \varphi d x \leq \int_{\Omega} f \varphi d x
$$

for every $\varphi \in C_{0}^{\infty}(\Omega), \varphi \geq 0$.

We define, for $k>0$, the truncation function at levels $\pm k$, that is,

$$
T_{k}(s)=\max \{\min \{s, k\},-k\} .
$$

We will also denote by $u^{+}, u^{-}$the positive and negative parts of $u$, i.e.,

$$
u^{+}=\max \{u, 0\}, \quad u^{-}=\max \{-u, 0\} .
$$

If $q \in(1, \infty)$, we will denote by $q^{\prime}$ its Hölder's conjugate exponent, that is, $q^{\prime}=\frac{q}{q-1}$. If $q \in[1, N)$, we will denote by $q^{*}$ its Sobolev conjugate exponent, that is, $q^{*}=\frac{q N}{N-q}$.

\section{LOCAL AND GLOBAL HÖLDER CONTINUity}

The basic starting point of our analysis is the following estimate.

Lemma 3.1. Assume (2.2), let $p>2, \lambda \geq 0$, and let $f$ belong to $L_{\text {loc }}^{q}(\Omega)$ for some $q \geq 1$. Let $u \in W_{\mathrm{loc}}^{1, p}(\Omega)$ be a subsolution of (2.1) in the sense of distributions, such that $\lambda u^{-} \in L_{\text {loc }}^{q}(\Omega)$. Then, for every pair of concentric balls $B_{\rho} \subset B_{R} \subset \Omega$, we have

$$
\int_{B_{\rho}}|\nabla u|^{p} d x+\lambda \int_{B_{\rho}} u^{+} d x \leq K \frac{R^{N}}{(R-\rho)^{\gamma}}
$$

where $\gamma=\max \left\{\frac{N}{q}, p^{\prime}\right\}$ and $K$ is a constant which depends on $\beta, p, q, N$, $\operatorname{diam}(\Omega)$, $\left\|f+\lambda u^{-}\right\|_{L^{q}\left(B_{R}\right)}$.

Proof. Let $C$ denote a generic constant, possibly depending on $\beta, N, p, q$. Let $\eta \in C^{1}$ be a cut-off function such that $0 \leq \eta \leq 1, \eta \equiv 1$ on $B_{\rho}, \eta \equiv 0$ outside $B_{R}$, and $|\nabla \eta| \leq \frac{C}{R-\rho}$. Multiplying (2.1) by $\eta^{2}$ and integrating by parts we have

$$
\int_{\Omega}|\nabla u|^{p} \eta^{2} d x+\lambda \int_{\Omega} u \eta^{2} d x \leq \int_{\Omega} f \eta^{2} d x-2 \int_{\Omega} a(x, u, \nabla u) \nabla \eta \eta d x
$$


which yields, using (2.2),

$$
\int_{\Omega}|\nabla u|^{p} \eta^{2} d x+\lambda \int_{\Omega} u^{+} \eta^{2} d x \leq \int_{\Omega}\left(f+\lambda u^{-}\right) \eta^{2} d x+2 \beta \int_{\Omega}(1+|\nabla u|) \eta|\nabla \eta| d x .
$$

Then the properties of $\eta$ and Young's inequality imply

$$
\begin{aligned}
2 \beta \int_{\Omega}(1+|\nabla u|) \eta & |\nabla \eta| d x \\
& \leq \frac{1}{2} \int_{\Omega}|\nabla u|^{p} \eta^{2} d x+C \int_{\Omega} \eta|\nabla \eta| d x+C \int_{\Omega} \eta^{\frac{p-2}{p-1}}|\nabla \eta|^{p^{\prime}} d x \\
& \leq \frac{1}{2} \int_{\Omega}|\nabla u|^{p} \eta^{2} d x+C \frac{R^{N}}{R-\rho}+C \frac{R^{N}}{(R-\rho)^{p^{\prime}}} \\
& \leq \frac{1}{2} \int_{\Omega}|\nabla u|^{p} \eta^{2} d x+C\left[\operatorname{diam}(\Omega)^{p^{\prime}-1}+1\right] \frac{R^{N}}{(R-\rho)^{p^{\prime}}} .
\end{aligned}
$$

On the other hand, by Hölder's inequality,

$$
\int_{\Omega}\left(f+\lambda u^{-}\right) \eta^{2} d x \leq\left\|f+\lambda u^{-}\right\|_{L^{q}\left(B_{R}\right)}\left|B_{R}\right|^{1-\frac{1}{q}} \leq C\left\|f+\lambda u^{-}\right\|_{L^{q}\left(B_{R}\right)} R^{N-\frac{N}{q}} .
$$

Therefore, (3.2) implies

$$
\begin{aligned}
\int_{B_{\rho}}|\nabla u|^{p} d x+\lambda \int_{B_{\rho}} u^{+} d x \leq C\left\|f+\lambda u^{-}\right\|_{L^{q}\left(B_{R}\right)} R^{N-\frac{N}{q}} & \\
& +C\left[\operatorname{diam}(\Omega)^{p^{\prime}-1}+1\right] \frac{R^{N}}{(R-\rho)^{p^{\prime}}} .
\end{aligned}
$$

In particular, we deduce (3.1).

The main consequence of estimate (3.1) is the local Hölder continuity of $u$. In the proof below, we also give a (uniform) estimate for the Hölder seminorm on any ball $B \subset \Omega$.

Theorem 3.1. Assume (2.2), let $p>2, \lambda \geq 0$, and let $f$ belong to $L^{q}(\Omega)$ for some $q>\frac{N}{p}$. Let $u \in W_{\mathrm{loc}}^{1, p}(\Omega)$ be such that $\lambda u^{-} \in L^{q}(\Omega)$ and such that the inequality (2.1) holds in the sense of distributions.

Then $u$ is locally Hölder continuous and satisfies, for every ball $B \subset \Omega$,

$$
|u(x)-u(y)| \leq K|x-y|^{\alpha}, \quad \forall x, y \in B,
$$

where $\alpha=\min \left(1-\frac{N}{p q}, 1-\frac{1}{p-1}\right)$ and $K$ depends only on $p, q, N, \beta, \operatorname{diam}(\Omega)$ and $\left\|f+\lambda u^{-}\right\|_{L^{q}(\Omega)}$.

Proof.

Step 1. Let $x_{0} \in \Omega$ and $B_{r}\left(x_{0}\right)$ be a ball such that $B_{2 r}\left(x_{0}\right) \subset \Omega$. It follows from Lemma 3.1 that

$$
\int_{B_{r}}|\nabla u|^{p} d x \leq K r^{N-\gamma},
$$

where $\gamma=\max \left(\frac{N}{q}, p^{\prime}\right)$ and $K$ depends on $p, q, N, \beta, \operatorname{diam}(\Omega)$ and $\left\|f+\lambda u^{-}\right\|_{L^{q}(\Omega)}$. Since we have

$$
\int_{B_{r}}|\nabla u| d x \leq\left(\int_{B_{r}}|\nabla u|^{p} d x\right)^{\frac{1}{p}}\left|B_{r}\left(x_{0}\right)\right|^{1-\frac{1}{p}}
$$


we deduce that $u$ satisfies, for some different constant still denoted by $K$,

$$
\int_{B_{r}}|\nabla u| d x \leq K r^{N-\frac{\gamma}{p}} .
$$

In particular, if $B_{R}$ is any ball such that $B_{2 R} \subset \Omega$, the same property will be true for any other ball $B_{r}$ contained in $B_{R}$, so that (3.3) will hold for every $B_{r} \subset B_{R}$. By Theorem 7.19 in 9 , we conclude that $u$ is Hölder continuous in $B_{R}$ with exponent $\alpha=1-\frac{\gamma}{p}=\min \left(1-\frac{N}{q p}, 1-\frac{1}{p-1}\right)$ and

$$
|u(x)-u(y)| \leq K|x-y|^{\alpha}
$$

for every $x, y \in B_{R}$. In particular, we have obtained that (3.4) holds for any couple of points $x, y$ which belong to some ball $B_{R}$ such that $B_{2 R} \subset \Omega$.

Step 2. Now let $B=B_{R}\left(x_{0}\right)$ be any ball such that $B \subset \Omega$. We are going to prove that (3.4) holds for every $x, y \in B$ with a (possibly different) constant $K$ independent on $B$.

Consider first the case when $x$ and $y$ lie on the same ray, say $x=x_{0}+s \sigma_{0}$ and $y=x_{0}+t \sigma_{0}$ for some $\sigma_{0}$ such that $\left|\sigma_{0}\right|=1$ and some real numbers $s, t$ with, say, $s>t$. Take the sequence of points $z_{n}=x-\frac{s-t}{2^{n}} \sigma_{0}$, so that $z_{0}=y$ and $z_{n} \rightarrow x$. It is not difficult to realize that we can apply (3.4) to any couple of points $z_{n}, z_{n-1}$; indeed, for every $\varepsilon>0$, these two points belong to the ball $B \frac{\left|z_{n}-z_{n-1}\right|}{2}+\varepsilon\left(\frac{z_{n}+z_{n-1}}{2}\right)$ which has center the mid-point $\frac{z_{n}+z_{n-1}}{2}$ and radius equal to $\frac{\left|z_{n}-z_{n-1}\right|}{2}+\varepsilon=\frac{s-t}{2^{n+1}}+\varepsilon$, and the same ball of twice a radius is still contained in $B$ for $\varepsilon$ small enough. Therefore we have

$$
\left|u\left(z_{n}\right)-u\left(z_{n-1}\right)\right| \leq K\left|z_{n}-z_{n-1}\right|^{\alpha} \quad \forall n \geq 1,
$$

hence, recalling that $\left|z_{n}-z_{n-1}\right|=\frac{|s-t|}{2^{n}}=\frac{|x-y|}{2^{n}}$ we get

$$
\left|u\left(z_{n}\right)-u(y)\right| \leq \sum_{k=1}^{n}\left|u\left(z_{k}\right)-u\left(z_{k-1}\right)\right| \leq K|x-y|^{\alpha} \sum_{k=1}^{n} \frac{1}{2^{k \alpha}},
$$

which implies, when $n \rightarrow \infty$ (we use here the continuity of $u$, which is a consequence of Step 1)

$$
|u(x)-u(y)| \leq \frac{K}{1-2^{-\alpha}}|x-y|^{\alpha} .
$$

Now take any $x, y \in B$. We denote by $d(x), d(y)$ the distance of the two points to the boundary of the ball, and by $R$ the radius. In view of (3.4), it is enough to discuss the case when $d(x), d(y)<\frac{R}{2}$.

Moreover, observe that, if $\max \{d(x), d(y)\}>\frac{3}{2}|x-y|$, we can also apply (3.4) to $x, y$. Indeed, we have $x, y \in B_{\frac{|x-y|}{2}}\left(\frac{x+y}{2}\right)$ and in this case the ball with double radius, which is $B_{|x-y|}\left(\frac{x+y}{2}\right)$, must be contained in $B$ (since $\max (d(x), d(y)) \leq$ $\left.d\left(\frac{x+y}{2}\right)+\frac{|x-y|}{2}\right)$.

We are left with the case that $\max (d(x), d(y)) \leq \frac{3}{2}|x-y|$; then consider two points $\bar{x}, \bar{y}$ such that $\bar{x}=x-d \nu(x)$ and $\bar{y}=y-d \nu(y)$ with $d=\min \left(\frac{R}{2}, \frac{3}{2}|x-y|\right)$ and $\nu(x)=\frac{x-x_{0}}{\left|x-x_{0}\right|}$. We first claim that (3.4) applies to $\bar{x}, \bar{y}$ : indeed, we have

$$
d(\bar{x})=d(x)+d, \quad d(\bar{y})=d(y)+d, \quad|\bar{x}-\bar{y}| \leq|x-y| .
$$


Now, if $d=\frac{3}{2}|x-y|$, this means that

$$
\max (d(\bar{x}), d(\bar{y}))>\frac{3}{2}|x-y| \geq \frac{3}{2}|\bar{x}-\bar{y}|
$$

and we are in the preceding case, while if $d=\frac{R}{2}$ this means that both $\bar{x}$ and $\bar{y}$ belong to $B_{\frac{R}{2}}$ and again (3.4) can be applied. Therefore in any case we can use (3.4) to get

$$
|u(\bar{x})-u(\bar{y})| \leq K|\bar{x}-\bar{y}|^{\alpha} \leq K|x-y|^{\alpha} .
$$

On the other hand, for points which are on the same rays we have

$$
|u(\bar{x})-u(x)| \leq \frac{K}{1-2^{-\alpha}}|x-\bar{x}|^{\alpha} \leq \frac{K}{1-2^{-\alpha}}\left(\frac{3}{2}\right)^{\alpha}|x-y|^{\alpha},
$$

and so

$$
|u(\bar{y})-u(y)| \leq \frac{K}{1-2^{-\alpha}}\left(\frac{3}{2}\right)^{\alpha}|x-y|^{\alpha} .
$$

Therefore we conclude

$$
|u(x)-u(y)| \leq \frac{2 K}{1-2^{-\alpha}}\left(\frac{3}{2}\right)^{\alpha}|x-y|^{\alpha}+K|x-y|^{\alpha}=\tilde{K}|x-y|^{\alpha}
$$

for $\tilde{K}=\left(\frac{2}{1-2^{-\alpha}}\left(\frac{3}{2}\right)^{\alpha}+1\right) K$.

Proof of Theorem 1.1. The proof follows from Theorem 3.1 applying Lemma 2.6 in [8].

Remark 3.1. The estimate (3.1) holds true under the weaker assumption that $f+$ $\lambda u^{-} \in \mathcal{L}^{1, \frac{N}{q}}(\Omega)$, i.e. the Morrey space of functions $g$ such that

$$
\int_{B_{r}}|g| \leq C r^{N\left(1-\frac{1}{q}\right)} \quad \text { for every ball } B_{r} \subset \Omega .
$$

As a consequence, the conclusion of Theorem 3.1 and Theorem 1.1 hold true in this more general case. Notice that every $g \in L^{q}(\Omega)$ clearly satisfies the above estimate after Hölder's inequality.

Remark 3.2. The result of Theorem 1.1 is optimal as far as the Hölder regularity of $u$ is concerned. Similarly as in [8], one can observe such optimality through the simplest example, namely taking $a(x, s, \xi)=\xi$ and $u(x)=|x|^{\alpha}$.

If we fix $q$ such that $\frac{N}{p}<q<\frac{N}{p^{\prime}}$, and $\alpha=1-\frac{N}{p q}$, then $u$ satisfies (2.1) for some $f$ belonging to the Morrey space $\mathcal{L}^{1, \frac{N}{q}}(\Omega)$, showing that the Hölder class (of order $1-\frac{N}{p q}$ ) cannot be improved. With a slight variation the same can be done with $f$ in a Lebesgue space, taking e.g. $u(x)=\left.|x|^{\alpha}|\ln | x\right|^{\gamma}$ for some $\gamma$ suitably chosen; in this case $u$ satisfies (2.1) with $f \in L^{q}(\Omega)$, showing again that the exponent of the Hölder regularity cannot be improved.

When $q \geq \frac{N}{p^{\prime}}$, the subsolutions will belong to $C^{\alpha}(\Omega)$ with $\alpha=\frac{p-2}{p-1}$. In this case an example of the optimality of Theorem 1.1 can be obtained even with $f \equiv 0$. Indeed, one can take $u(x)=c_{0}\left(|x|^{\frac{p-2}{p-1}}-1\right)$ which, for a suitable choice of $c_{0}$, satisfies (in the distributional sense)

$$
\left\{\begin{array}{l}
-\Delta u+|\nabla u|^{p}=0 \quad \text { in } \Omega, \\
u \in W_{0}^{1, p}(\Omega),
\end{array}\right.
$$

where $\Omega=B_{1}(0)$. 
It is worth noticing, in this example, that $u$ is a distributional solution of the equation, and not only a subsolution. On one hand, the regularity is therefore optimal even in the class of distributional solutions. On the other hand, observe that the uniqueness fails even for bounded, Hölder continuous distributional solutions. On the contrary, the above function $u(x)$ is not a viscosity solution of (3.5) (it does not satisfy the condition of a supersolution at the point $x=0$ ). This is consistent with the uniqueness of viscosity solutions (see [2]). It is interesting to observe that uniqueness really depends here on the formulation (viscosity rather than distributional) within the same class of Hölder continuous functions.

Remark 3.3. It is easy to check that, in Theorems 1.1 and 3.1, a datum in divergence form can be added, without any substantial change in the proof. More precisely, if we assume that the vector valued function $a$ satisfies, instead of (1.2), the weaker condition

$$
|a(x, s, \xi)| \leq g(x)+\beta|\xi|,
$$

where $\beta>0$ and

$$
g(x) \in L^{\sigma}(\Omega), \text { with } \sigma>\frac{N}{p-1},
$$

then the statements of Theorems 1.1 and 3.1 remain the same, with

$$
\alpha=1-\max \left(\frac{N}{p q}, \frac{N+\sigma}{p \sigma}, \frac{1}{p-1}\right) .
$$

\section{Local REgularity in Lebesgue SPACES}

In this section, we turn our attention to estimating the local norm of $u$ rather than its oscillation. Of course this makes sense only in the case $\lambda>0$ (if $\lambda=0$, (2.1) may be invariant by adding a constant to $u$ ). We start with the case where the datum $f$ belongs to $L_{\text {loc }}^{q}(\Omega)$, with $q$ below the critical value $\frac{N}{p}$.

Theorem 4.1. Assume (2.2), let $2<p<N, \lambda>0$ and let $f$ belong to $L_{\mathrm{loc}}^{q}(\Omega)$ for some $q<\frac{N}{p}$. Let $u \in W_{\mathrm{loc}}^{1, p}(\Omega)$ be a subsolution of (2.1) in the sense of distributions. Then $u^{+} \in L_{\text {loc }}^{s}(\Omega)$ with $s=\frac{N p q}{N-p q}$. Moreover, for every pair of concentric balls $B_{\rho} \subset B_{R} \subset \Omega$, we have

$$
\left\|u^{+}\right\|_{L^{s}\left(B_{\rho}\right)} \leq K
$$

where $K$ depends on $\beta, p, q, N, \lambda^{-1}, \rho, R,\|f\|_{L^{q}\left(B_{R}\right)},\left\|u^{+}\right\|_{L^{1}\left(B_{R}\right)}$ and, if $1 \leq q<$ $\frac{N(p-1)}{N(p-2)+p}$, it depends on $\left\|\left|\nabla u^{+}\right|\right\|_{L^{p}\left(B_{R}\right)}$ as well.

Proof. Let $C$ denote a generic constant, possibly depending on $\beta, N, p, q$. Let us take a cut-off function $\eta \in C_{c}^{\infty}\left(B_{R}\right)$ such that $0 \leq \eta \leq 1, \eta=1$ on $B_{\rho}$, $|\nabla \eta| \leq \frac{C}{R-\rho}$. We start by assuming that $q \geq \frac{N(p-1)}{N(p-2)+p}$. For $\gamma, \alpha>0$ (to be chosen below, depending only on $p, q, N)$, we take $\varphi=T_{k}\left(u^{+}\right)^{\gamma p} \eta^{\alpha p}$ as the test function 
in (2.3). Notice that the test function vanishes on the set where $u \leq 0$. We obtain

$$
\begin{gathered}
\int_{\Omega}|\nabla u|^{p} T_{k}\left(u^{+}\right)^{\gamma p} \eta^{\alpha p} d x+\lambda \int_{\Omega} u^{+} T_{k}\left(u^{+}\right)^{\gamma p} \eta^{\alpha p} d x \\
\leq C \int_{\Omega}(1+|\nabla u|)\left|\nabla u^{+}\right| T_{k}\left(u^{+}\right)^{\gamma p-1} \eta^{\alpha p} d x \\
+C \int_{\Omega}(1+|\nabla u|)|\nabla \eta| T_{k}\left(u^{+}\right)^{\gamma p} \eta^{\alpha p-1} d x \\
+\int_{\Omega}|f| T_{k}\left(u^{+}\right)^{\gamma p} \eta^{\alpha p} d x,
\end{gathered}
$$

where the constant $C$ depends on $p, q, N$. Using Young's inequality, for every $\varepsilon>0$ (which will be chosen conveniently small later), we can write

$$
\begin{aligned}
\int_{\Omega}(1+|\nabla u|)\left|\nabla u^{+}\right| & T_{k}\left(u^{+}\right)^{\gamma p-1} \eta^{\alpha p} d x \leq \varepsilon \int_{\Omega}|\nabla u|^{p} T_{k}\left(u^{+}\right)^{\gamma p} \eta^{\alpha p} d x \\
& +C_{\varepsilon} \int_{\Omega} T_{k}\left(u^{+}\right)^{\gamma p-p^{\prime}} \eta^{\alpha p} d x+C_{\varepsilon} \int_{\Omega} T_{k}\left(u^{+}\right)^{\gamma p-\frac{p}{p-2}} \eta^{\alpha p} d x .
\end{aligned}
$$

We will check later that $\gamma$ satisfies $\gamma \geq \frac{1}{p-2}$ provided $q \geq \frac{N(p-1)}{N(p-2)+p}$. Then, since $0 \leq \gamma p-\frac{p}{p-2}<\gamma p-p^{\prime}<\gamma p+1$, we use Young's inequality once more, to obtain

$$
\begin{aligned}
\int_{\Omega}(1+|\nabla u|)\left|\nabla u^{+}\right| T_{k}\left(u^{+}\right)^{\gamma p-1} \eta^{\alpha p} d x & \leq \varepsilon \int_{\Omega}|\nabla u|^{p} T_{k}\left(u^{+}\right)^{\gamma p} \eta^{\alpha p} d x \\
& +\varepsilon \int_{\Omega} T_{k}\left(u^{+}\right)^{\gamma p+1} \eta^{\alpha p} d x+C_{\varepsilon}\left|B_{R}\right| .
\end{aligned}
$$

Similarly, we estimate

$$
\begin{aligned}
& \int_{\Omega}(1+|\nabla u|)|\nabla \eta| T_{k}\left(u^{+}\right)^{\gamma p} \eta^{\alpha p-1} d x \\
& \leq \varepsilon \int_{\Omega} T_{k}\left(u^{+}\right)^{\gamma p+1} \eta^{\alpha p} d x+C_{\varepsilon} \int_{\Omega}|\nabla \eta|^{\gamma p+1} \eta^{\alpha p-(\gamma p+1)} d x \\
& \quad+\varepsilon \int_{\Omega}|\nabla u|^{p} T_{k}\left(u^{+}\right)^{\gamma p} \eta^{\alpha p} d x+C_{\varepsilon} \int_{\Omega}|\nabla \eta|^{p^{\prime}} T_{k}\left(u^{+}\right)^{\gamma p} \eta^{\alpha p-p^{\prime}} d x
\end{aligned}
$$

and using again, in the last integral, Young's inequality with exponent $\gamma p+1$, we get

$$
\begin{aligned}
\int_{\Omega}(1+ & |\nabla u|)|\nabla \eta| T_{k}\left(u^{+}\right)^{\gamma p} \eta^{\alpha p-1} d x \\
& \leq \varepsilon \int_{\Omega} T_{k}\left(u^{+}\right)^{\gamma p+1} \eta^{\alpha p} d x+\varepsilon \int_{\Omega}|\nabla u|^{p} T_{k}\left(u^{+}\right)^{\gamma p} \eta^{\alpha p} d x \\
& +C_{\varepsilon} \int_{\Omega}|\nabla \eta|^{\gamma p+1} \eta^{\alpha p-(\gamma p+1)} d x+C_{\varepsilon} \int_{\Omega}|\nabla \eta|^{p^{\prime}(\gamma p+1)} \eta^{\alpha p-p^{\prime}(\gamma p+1)} d x .
\end{aligned}
$$


If we choose $\varepsilon$ small and $\alpha$ sufficiently large, so that $\alpha p \geq p^{\prime}(\gamma p+1)>(\gamma p+1)$, using (4.3) and (4.4) we deduce from (4.2):

$$
\begin{gathered}
\frac{1}{2} \int_{\Omega}|\nabla u|^{p} T_{k}\left(u^{+}\right)^{\gamma p} \eta^{\alpha p} d x+\frac{\lambda}{2} \int_{\Omega} u^{+} T_{k}\left(u^{+}\right)^{\gamma p} \eta^{\alpha p} d x \\
\leq C\left(\rho, R, \lambda^{-1}\right)+\int_{\Omega}|f| T_{k}\left(u^{+}\right)^{\gamma p} \eta^{\alpha p} d x
\end{gathered}
$$

where $C\left(\rho, R, \lambda^{-1}\right)$ depends now on $\rho, R$ and $\lambda^{-1}$ as well. We now use Sobolev's inequality, which yields

$$
\begin{aligned}
& \left(\int_{\Omega}\left|T_{k}\left(u^{+}\right)^{\gamma+1} \eta^{\alpha}\right|^{p^{*}} d x\right)^{\frac{p}{p^{*}}} \leq C \int_{\Omega}\left|\nabla\left(T_{k}\left(u^{+}\right)^{\gamma+1} \eta^{\alpha}\right)\right|^{p} d x \\
& \quad \leq C \int_{\Omega}|\nabla u|^{p} T_{k}\left(u^{+}\right)^{\gamma p} \eta^{\alpha p} d x+C \int_{\Omega} T_{k}\left(u^{+}\right)^{(\gamma+1) p}|\nabla \eta|^{p} \eta^{(\alpha-1) p} d x .
\end{aligned}
$$

By interpolation, since $1<(\gamma+1) p<(\gamma+1) p^{*}$, we get

$$
\begin{aligned}
& \int_{\Omega} T_{k}\left(u^{+}\right)^{(\gamma+1) p}|\nabla \eta|^{p} \eta^{(\alpha-1) p} d x \\
& \quad \leq\left(\int_{\Omega} T_{k}\left(u^{+}\right)^{(\gamma+1) p^{*}} \eta^{\alpha p^{*}} d x\right)^{\theta}\left(\int_{\Omega} T_{k}\left(u^{+}\right)|\nabla \eta|^{\frac{p}{1-\theta}} \eta^{\frac{(\alpha-1) p-\alpha p^{*} \theta}{1-\theta}} d x\right)^{1-\theta}
\end{aligned}
$$

where $(\gamma+1) p=\theta(\gamma+1) p^{*}+1-\theta$, i.e.

$$
\theta=\frac{(\gamma+1) p-1}{(\gamma+1) p^{*}-1}
$$

Note that $\theta<\frac{p}{p^{*}}$, in particular, $(\alpha-1) p-\alpha p^{*} \theta>0$ provided $\alpha$ is sufficiently large.

We deduce that, using Young's inequality,

$$
\begin{aligned}
\int_{\Omega} T_{k}\left(u^{+}\right)^{(\gamma+1) p} \mid \nabla & \left.\eta\right|^{p} \eta^{(\alpha-1) p} d x \\
& \leq \varepsilon\left(\int_{\Omega} T_{k}\left(u^{+}\right)^{(\gamma+1) p^{*}} \eta^{\alpha p^{*}} d x\right)^{\frac{p}{p^{*}}}+C_{\varepsilon}\left(\rho, R,\left\|u^{+}\right\|_{L^{1}\left(B_{R}\right)}\right)
\end{aligned}
$$

and therefore (4.6) implies, for a suitable choice of $\varepsilon$,

$$
\begin{aligned}
\left(\int_{\Omega}\left|T_{k}\left(u^{+}\right)^{\gamma+1} \eta^{\alpha}\right|^{p^{*}} d x\right)^{\frac{p}{p^{*}}} & \\
& \leq C \int_{\Omega}|\nabla u|^{p} T_{k}\left(u^{+}\right)^{\gamma p} \eta^{\alpha p} d x+C\left(\rho, R,\left\|u^{+}\right\|_{L^{1}\left(B_{R}\right)}\right) .
\end{aligned}
$$

Then, we obtain from (4.5)

$$
\begin{aligned}
\left(\int_{\Omega}\left|T_{k}\left(u^{+}\right)^{\gamma+1} \eta^{\alpha}\right|^{p^{*}} d x\right)^{\frac{p}{p^{*}}} & +\lambda \int_{\Omega} u^{+} T_{k}\left(u^{+}\right)^{\gamma p} \eta^{\alpha p} d x \\
\leq & C\left(\rho, R, \lambda^{-1},\left\|u^{+}\right\|_{L^{1}\left(B_{R}\right)}\right)+\int_{\Omega}|f| T_{k}\left(u^{+}\right)^{\gamma p} \eta^{\alpha p} d x
\end{aligned}
$$


which yields, using Hölder's inequality in the right-hand side,

$$
\begin{aligned}
& \left(\int_{\Omega}\left|T_{k}\left(u^{+}\right)^{\gamma+1} \eta^{\alpha}\right|^{p^{*}} d x\right)^{\frac{p}{p^{*}}}+\lambda \int_{\Omega} u^{+} T_{k}\left(u^{+}\right)^{\gamma p} \eta^{\alpha p} d x \\
& \quad \leq C\left(\rho, R, \lambda^{-1},\left\|u^{+}\right\|_{L^{1}\left(B_{R}\right)}\right)+\|f\|_{L^{q}\left(B_{R}\right)}\left(\int_{\Omega} T_{k}\left(u^{+}\right)^{\gamma p q^{\prime}} \eta^{\alpha p q^{\prime}} d x\right)^{\frac{1}{q^{\prime}}} .
\end{aligned}
$$

Now we choose $\gamma$ so that $(\gamma+1) p^{*}=\gamma p q^{\prime}$, i.e.

$$
\gamma=\frac{N(q-1)}{N-p q} .
$$

Note that $\gamma>0 \Longleftrightarrow q^{\prime} p>p^{*} \Longleftrightarrow q<\frac{N}{p}$, and that the condition $\gamma \geq \frac{1}{p-2}$ is equivalent to $q \geq \frac{N(p-1)}{N(p-2)+p}$. We also have $\alpha p q^{\prime}>\alpha p^{*}$, hence

$$
\left(\int_{\Omega} T_{k}\left(u^{+}\right)^{\gamma p q^{\prime}} \eta^{\alpha p q^{\prime}} d x\right)^{\frac{1}{q^{\prime}}} \leq\left(\int_{\Omega}\left|T_{k}\left(u^{+}\right)^{\gamma+1} \eta^{\alpha}\right|^{p^{*}} d x\right)^{\frac{1}{q^{\prime}}}
$$

and since $\frac{1}{q^{\prime}}<\frac{p}{p^{*}}$ and $s=\frac{N p q}{N-p q}=(\gamma+1) p^{*}$, letting $k \rightarrow \infty$ we conclude with the estimate

$$
\left\|u^{+}\right\|_{L^{s}\left(B_{\rho}\right)} \leq K
$$

where $K=K\left(\beta, p, q, N, \rho, R, \lambda^{-1},\|f\|_{L^{q}\left(B_{R}\right)},\left\|u^{+}\right\|_{L^{1}\left(B_{R}\right)}\right)$.

In order to deal with the whole range of values of $q$, including $q<\frac{N(p-1)}{N(p-2)+p}$, we slightly modify the above argument. We now take $\varphi=\left[\left(1+T_{k}\left(u^{+}\right)\right)^{\gamma p}-1\right] \eta^{\alpha p}$ as the test function in (2.3), and we get

$$
\begin{gathered}
\int_{\Omega}|\nabla u|^{p}\left[\left(1+T_{k}\left(u^{+}\right)\right)^{\gamma p}-1\right] \eta^{\alpha p} d x+\lambda \int_{\Omega} u^{+}\left[\left(1+T_{k}\left(u^{+}\right)\right)^{\gamma p}-1\right] \eta^{\alpha p} d x \\
\leq C \int_{\Omega}(1+|\nabla u|)\left|\nabla u^{+}\right|\left(1+T_{k}\left(u^{+}\right)\right)^{\gamma p-1} \eta^{\alpha p} d x \\
+C \int_{\Omega}(1+|\nabla u|)|\nabla \eta|\left(1+T_{k}\left(u^{+}\right)\right)^{\gamma p} \eta^{\alpha p-1} d x \\
+\int_{\Omega}|f|\left(1+T_{k}\left(u^{+}\right)\right)^{\gamma p} \eta^{\alpha p} d x .
\end{gathered}
$$

We now estimate the first term in the right-hand side as

$$
\begin{gathered}
\int_{\Omega}(1+|\nabla u|)\left|\nabla u^{+}\right|\left(1+T_{k}\left(u^{+}\right)\right)^{\gamma p-1} \eta^{\alpha p} d x \leq \varepsilon \int_{\Omega}|\nabla u|^{p}\left(1+T_{k}\left(u^{+}\right)\right)^{\gamma p} \eta^{\alpha p} d x \\
+C_{\varepsilon} \int_{\Omega}\left(1+T_{k}\left(u^{+}\right)\right)^{\gamma p-p^{\prime}} \eta^{\alpha p} d x+C_{\varepsilon} \int_{\Omega}\left(1+T_{k}\left(u^{+}\right)\right)^{\gamma p-\frac{p}{p-2}} \eta^{\alpha p} d x \\
\leq \varepsilon \int_{\Omega}|\nabla u|^{p}\left(1+T_{k}\left(u^{+}\right)\right)^{\gamma p} \eta^{\alpha p} d x+C_{\varepsilon} \int_{\Omega}\left(1+T_{k}\left(u^{+}\right)\right)^{\gamma p} \eta^{\alpha p} d x
\end{gathered}
$$


obtaining then, after Young's inequality,

$$
\begin{array}{rl}
\int_{\Omega}(1+|\nabla u|)\left|\nabla u^{+}\right|\left(1+T_{k}\left(u^{+}\right)\right)^{\gamma p-1} \eta^{\alpha p} & d x \leq \varepsilon \int_{\Omega}|\nabla u|^{p}\left(1+T_{k}\left(u^{+}\right)\right)^{\gamma p} \eta^{\alpha p} d x \\
& +\varepsilon \int_{\Omega} T_{k}\left(u^{+}\right)^{\gamma p+1} \eta^{\alpha p} d x+C_{\varepsilon}\left|B_{R}\right| .
\end{array}
$$

The second term in the right-hand side of (4.7) is dealt with in a similar way as in the previous case. Then we obtain the inequality

$$
\begin{gathered}
\frac{1}{2} \int_{\Omega}|\nabla u|^{p}\left(1+T_{k}\left(u^{+}\right)\right)^{\gamma p} \eta^{\alpha p} d x+\frac{\lambda}{2} \int_{\Omega} u^{+}\left(1+T_{k}\left(u^{+}\right)\right)^{\gamma p} \eta^{\alpha p} d x \\
\leq C\left(\rho, R, \lambda^{-1}\right)+\int_{\Omega}|f|\left(1+T_{k}\left(u^{+}\right)\right)^{\gamma p} \eta^{\alpha p} d x \\
+\int_{\Omega}\left|\nabla u^{+}\right|^{p} \eta^{\alpha p} d x+\lambda \int_{\Omega} u^{+} \eta^{\alpha p} d x .
\end{gathered}
$$

Henceforth, we proceed as before, using Sobolev's inequality in the left-hand side and Hölder's inequality in the term with $f$. With the choice $(\gamma+1) p^{*}=\gamma p q^{\prime}$ made before, we obtain therefore

$$
\begin{aligned}
& \left(\int_{\Omega}\left|T_{k}\left(u^{+}\right)^{\gamma+1} \eta^{\alpha}\right|^{p^{*}} d x\right)^{\frac{p}{p^{*}}}+\lambda \int_{\Omega} u T_{k}\left(u^{+}\right)^{\gamma p} \eta^{\alpha p} d x \\
& \leq C\left(\rho, R, \lambda^{-1},\left\|u^{+}\right\|_{L^{1}\left(B_{R}\right)}\right)+\|f\|_{L^{q}\left(B_{R}\right)}\left(\int_{\Omega} T_{k}\left(u^{+}\right)^{(\gamma+1) p^{*}} \eta^{\alpha p^{*}} d x\right)^{\frac{1}{q^{\prime}}} \\
& +\|f\|_{L^{1}\left(B_{R}\right)}+\int_{\Omega}\left|\nabla u^{+}\right|^{p} \eta^{\alpha p} d x+\lambda \int_{\Omega} u^{+} \eta^{\alpha p} d x .
\end{aligned}
$$

Letting $k$ go to infinity, we conclude with the estimate

$$
\left\|u^{+}\right\|_{L^{s}\left(B_{\rho}\right)} \leq K
$$

where $K=K\left(\beta, p, q, N, \rho, R, \lambda^{-1},\|f\|_{L^{q}\left(B_{R}\right)},\left\|u^{+}\right\|_{L^{1}\left(B_{R}\right)},\left\|\left|\nabla u^{+}\right|\right\|_{L^{p}\left(B_{R}\right)}\right)$.

Remark 4.1. We can always estimate the $L^{1}$-norm of $u^{+}$in terms of the $L^{1}$-norm of $\left|\nabla u^{+}\right|^{p}$. Indeed, taking $\varphi=T_{1}\left(u^{+}\right) \eta^{2}$ as the test function, and using (2.2), we have

$$
\begin{aligned}
& \int_{\Omega}|\nabla u|^{p} T_{1}\left(u^{+}\right) \eta^{2} d x+\lambda \int_{\Omega} u^{+} T_{1}\left(u^{+}\right) \eta^{2} d x \leq \int_{\Omega}|f| T_{1}\left(u^{+}\right) \eta^{2} d x \\
& +\beta \int_{\Omega}(1+|\nabla u|)\left|\nabla T_{1}\left(u^{+}\right)\right| \eta^{2} d x+2 \beta \int_{\Omega}(1+|\nabla u|) T_{1}\left(u^{+}\right)|\nabla \eta| \eta d x
\end{aligned}
$$

which yields, by Young's inequality,

$$
\begin{gathered}
\frac{1}{2} \int_{\Omega}|\nabla u|^{p} T_{1}\left(u^{+}\right) \eta^{2} d x+\lambda \int_{\Omega} u^{+} T_{1}\left(u^{+}\right) \eta^{2} d x \leq \int_{\Omega}|f| \eta^{2} d x \\
+C \int_{\Omega}\left|\nabla u^{+}\right|^{p} \eta^{2} d x+C \int_{\Omega}|\nabla \eta|^{p^{\prime}} d x+C .
\end{gathered}
$$

Then we deduce

$$
\left\|u^{+}\right\|_{L^{1}\left(B_{\rho}\right)} \leq C\left(\beta, p, N, \lambda^{-1}, \rho, R,\left\|\left|\nabla u^{+}\right|\right\|_{L^{p}\left(B_{R}\right)}\right) .
$$


In particular, since the choice of balls is arbitrary, we deduce that estimate (4.1) holds true, for every $q<\frac{N}{p}$, with a constant only depending on $\left\|\left|\nabla u^{+}\right|\right\|_{L^{p}\left(B_{R}\right)}$ (beyond the usual parameters and constants).

Remark 4.2. If $p \geq N$, a similar result can be obtained with $s$ being any value such that $s>1$. Indeed, in this case we use Sobolev embedding of $W_{0}^{1, p}(\Omega)$ into $L^{r}(\Omega)$ which holds for every $r>1$. By proceeding as in the above proof (replacing $p^{*}$ with a generic $r>1$ ) we obtain the estimate for every possible $s>1$ with a constant $K$ depending on $s$ as well.

Remark 4.3. One can also treat the case where a datum in divergence form is present. More precisely, if we assume that the vector valued function $a(x, s, \xi)$ satisfies (3.6), with

$$
g(x) \in L_{\mathrm{loc}}^{\sigma}(\Omega), \quad \sigma=\frac{N q}{N-q},
$$

then it is easy to check that Theorem 4.1 continues to hold true, with the bound $K$ depending also on $\|g\|_{L^{\sigma}\left(B_{R}\right)}$.

We now prove an estimate of the local $L^{\infty}$-norm of $u$.

Theorem 4.2. Assume (2.2), let $p>2, \lambda>0$, and let $f$ belong to $L_{\text {loc }}^{q}(\Omega)$ for some $q>\frac{N}{p}$. Let $u \in W_{\mathrm{loc}}^{1, p}(\Omega)$ be a subsolution of (2.1) in the sense of distributions. Then we have $u^{+} \in L_{\mathrm{loc}}^{\infty}(\Omega)$ and, for every pair of concentric balls $B_{\rho} \subset B_{R} \subset \Omega$, we have

$$
\left\|u^{+}\right\|_{L^{\infty}\left(B_{r}\right)} \leq K
$$

where $K=K\left(\beta, p, q, N, \lambda^{-1}, \rho, R,\|f\|_{L^{q}\left(B_{R}\right)},\left\|u^{+}\right\|_{L^{1}\left(B_{R}\right)}\right)$.

Proof. First of all, observe that, by the previous result, $u$ belongs to $L_{\text {loc }}^{s}(\Omega)$ for all $s<\infty$, and that an estimate like (4.1) holds in terms of the $L^{1}$-norm of $u^{+}$in a slightly larger ball. Moreover, by the usual inclusions between Lebesgue spaces, one can always suppose that

$$
\frac{N}{p}<q \leq \frac{N}{p^{\prime}}
$$

Let us take $\varphi=v_{h, k}^{\frac{p}{p-2}} \eta^{p \alpha}$ as the test function in (2.3), where

$$
v_{h, k}=T_{h-k}\left(G_{k}\left(u^{+}\right)\right)
$$

with $G_{k}(s)=s-T_{k}(s), h>k>0, \alpha>0$ to be fixed later. As before, we denote by $\eta$ a cut-off function, $\eta \in C_{c}^{\infty}\left(B_{R}\right), 0 \leq \eta \leq 1, \eta=1$ on $B_{\rho},|\nabla \eta| \leq C(R-\rho)^{-1}$. In the following we set

$$
A(k, R)=\left\{x \in B_{R}: u(x)>k\right\} .
$$

Since $v_{h, k}=0$ in $\Omega \backslash A(k, R)$ we get

$$
\begin{gathered}
\int_{A(k, R)}|\nabla u|^{p} v_{h, k}^{\frac{p}{p-2}} \eta^{\alpha p} d x+\lambda \int_{A(k, R)} u v_{h, k}^{\frac{p}{p-2}} \eta^{\alpha p} d x \\
\leq C \int_{A(k, R)}(1+|\nabla u|)\left|\nabla u^{+}\right| v_{h, k}^{\frac{2}{p-2}} \eta^{\alpha p} d x \\
+C \int_{A(k, R)}(1+|\nabla u|)|\nabla \eta| v_{h, k}^{\frac{p}{p-2}} \eta^{\alpha p-1} d x+\int_{A(k, R)}|f| v_{h, k}^{\frac{p}{p-2}} \eta^{\alpha p} d x .
\end{gathered}
$$


Let us estimate the two terms in the second line above. By Young's inequality, for every $\varepsilon>0$, we have

$$
\begin{aligned}
& \int_{A(k, R)}(1+|\nabla u|)\left|\nabla u^{+}\right| v_{h, k}^{\frac{2}{p-2}} \eta^{\alpha p} d x \\
\leq & \varepsilon \int_{A(k, R)}|\nabla u|^{p} v_{h, k}^{\frac{p}{p-2}} \eta^{\alpha p} d x+C_{\varepsilon} \int_{A(k, R)} v_{h, k}^{\frac{p}{(p-2)(p-1)}} \eta^{\alpha p} d x+C_{\varepsilon} \int_{A(k, R)} \eta^{\alpha p} d x .
\end{aligned}
$$

Since $v_{h, k} \leq u^{+}$and $\frac{p}{(p-2)(p-1)}<1+\frac{p}{p-2}$, using Young's inequality once more, one obtains

$$
\begin{aligned}
& \int_{A(k, R)}(1+|\nabla u|)\left|\nabla u^{+}\right| v_{h, k}^{\frac{2}{p-2}} \eta^{\alpha p} d x \\
& \quad \leq \varepsilon \int_{A(k, R)}|\nabla u|^{p} v_{h, k}^{\frac{p}{p-2}} \eta^{\alpha p} d x+\varepsilon \int_{A(k, R)} u v_{h, k}^{\frac{p}{(p-2)}} \eta^{\alpha p} d x+C_{\varepsilon} \int_{A(k, R)} \eta^{\alpha p} d x .
\end{aligned}
$$

Similarly, we estimate

$$
\begin{aligned}
& \int_{A(k, R)}(1+|\nabla u|)|\nabla \eta| v_{h, k}^{\frac{p}{p-2}} \eta^{\alpha p-1} d x \\
& \leq \varepsilon \int_{A(k, R)} v_{h, k}^{\frac{p}{p-2}+1} \eta^{\alpha p} d x+C_{\varepsilon} \int_{A(k, R)}|\nabla \eta|^{1+\frac{p}{p-2}} \eta^{\alpha p-1-\frac{p}{p-2}} d x \\
& \quad+\varepsilon \int_{A(k, R)}|\nabla u|^{p} v_{h, k}^{\frac{p}{p-2}} \eta^{\alpha p} d x+C_{\varepsilon} \int_{A(k, R)}|\nabla \eta|^{p^{\prime}} v_{h, k}^{\frac{p}{p-2}} \eta^{\alpha p-p^{\prime}} d x .
\end{aligned}
$$

Using Young's inequality again in the last term with exponent $\frac{p}{p-2}+1$ and using $v_{h, k} \leq u^{+}$we obtain

$$
\begin{aligned}
\int_{A(k, R)} & (1+|\nabla u|)|\nabla \eta| v_{h, k}^{\frac{p}{p-2}} \eta^{\alpha p-1} d x \\
& \leq \varepsilon \int_{A(k, R)} u v_{h, k}^{\frac{p}{(p-2)}} \eta^{\alpha p} d x+C_{\varepsilon} \int_{A(k, R)}|\nabla \eta|^{1+\frac{p}{p-2}} \eta^{\alpha p-1-\frac{p}{p-2}} d x \\
& +\varepsilon \int_{A(k, R)}|\nabla u|^{p} v_{h, k}^{\frac{p}{p-2}} \eta^{\alpha p} d x+C_{\varepsilon} \int_{A(k, R)}|\nabla \eta|^{p^{\prime}\left(\frac{p}{p-2}+1\right)} \eta^{\alpha p-p^{\prime}\left(\frac{p}{p-2}+1\right)} d x .
\end{aligned}
$$

Choosing $\varepsilon$ suitably, we deduce from the above inequalities and (4.11)

$$
\begin{aligned}
\int_{A(k, R)}|\nabla u|^{p} v_{h, k}^{\frac{p}{p-2}} \eta^{\alpha p} d x+\lambda \int_{A(k, R)} u v_{h, k}^{\frac{p}{p-2}} \eta^{\alpha p} d x \\
\leq C \int_{A(k, R)} \eta^{\alpha p} d x+C \int_{A(k, R)}|\nabla \eta|^{\frac{2(p-1)}{p-2}} \eta^{\alpha p-\frac{2(p-1)}{p-2}} d x \\
\quad+C \int_{A(k, R)}|\nabla \eta|^{\frac{2 p}{p-2}} \eta^{\alpha p-\frac{2 p}{p-2}} d x+\int_{A(k, R)}|f| v_{h, k}^{\frac{p}{p-2}} \eta^{\alpha p} d x .
\end{aligned}
$$


ELLIPTIC EQUATIONS WITH SUPERQUADRATIC HAMILTONIAN

3031

Sobolev's inequality implies

$$
\begin{aligned}
& {\left[\int_{\Omega}\left(v_{h, k}^{\frac{p-1}{p-2}} \eta^{\alpha}\right)^{p^{*}} d x\right]^{\frac{p}{p^{*}}} \leq C \int_{\Omega}\left|\nabla\left(v_{h, k}^{\frac{p-1}{p-2}} \eta^{\alpha}\right)\right|^{p} d x} \\
& =C \int_{A(k, R)}|\nabla u|^{p} v_{h, k}^{\frac{p}{p-2}} \eta^{\alpha p} d x+C \int_{A(k, R)} v_{h, k}^{\frac{p(p-1)}{p-2}} \eta^{\alpha p-p}|\nabla \eta|^{p} d x
\end{aligned}
$$

(here we suppose $p<N$, otherwise one can replace $p^{*}$ with a conveniently high exponent), thus we can deduce from (4.12)

$$
\begin{aligned}
& {\left[\int_{\Omega}\left(v_{h, k}^{\frac{p-1}{p-2}} \eta^{\alpha}\right)^{p^{*}} d x\right]^{\frac{p}{p^{*}}} \leq C \int_{A(k, R)} \eta^{\alpha p} d x } \\
&+C \int_{A(k, R)}|\nabla \eta|^{\frac{2(p-1)}{p-2}} \eta^{\alpha p-\frac{2(p-1)}{p-2}} d x \\
&+C \int_{A(k, R)}|\nabla \eta|^{\frac{2 p}{p-2}} \eta^{\alpha p-\frac{2 p}{p-2}} d x+\int_{A(k, R)}|f| v_{h, k}^{\frac{p}{p-2}} \eta^{\alpha p} d x \\
&+\int_{A(k, R)} v_{h, k}^{\frac{p(p-1)}{p-2}} \eta^{\alpha p-p}|\nabla \eta|^{p} d x .
\end{aligned}
$$

Observing that $\frac{1}{q}+\frac{p^{\prime}}{p^{*}}<1$, and using Hölder's inequality with exponents $\left(q, \frac{p^{*}}{p^{\prime}}, \frac{1}{\frac{1}{q^{\prime}}-\frac{p^{\prime}}{p^{*}}}\right)$ we have

$$
\begin{aligned}
& \int_{A(k, R)}|f| v_{h, k}^{\frac{p}{p-2}} \eta^{\alpha p} d x \\
& \quad \leq\|f\|_{L^{q}\left(B_{R}\right)}\left(\int_{\Omega} v_{h, k}^{\frac{p-1}{p-2} p^{*}} \eta^{\alpha p^{*}} d x\right)^{\frac{p^{\prime}}{p^{*}}}\left(\int_{A(k, R)} \eta^{\alpha\left(p-p^{\prime}\right) \frac{q^{\prime} p^{*}}{p^{*}-q^{\prime} p^{\prime}}} d x\right)^{\frac{1}{q^{\prime}}-\frac{p^{\prime}}{p^{*}}}
\end{aligned}
$$

which implies, after Young's inequality with exponent $p-1$,

$$
\begin{aligned}
\int_{A(k, R)}|f| v_{h, k}^{\frac{p}{p-2}} \eta^{\alpha p} d x & \leq \varepsilon\left(\int_{\Omega} v_{h, k}^{\frac{p-1}{p-2} p^{*}} \eta^{\alpha p^{*}} d x\right)^{\frac{p}{p^{*}}} \\
& +C_{\varepsilon}\|f\|_{L^{q}\left(B_{R}\right)}^{\frac{p-1}{p-2}}\left(\int_{A(k, R)} \eta^{\alpha\left(p-p^{\prime}\right) \frac{q^{\prime} p^{*}}{p^{*}-q^{\prime} p^{\prime}}} d x\right)^{\left(\frac{1}{q^{\prime}}-\frac{p^{\prime}}{p^{*}}\right) \frac{p-1}{p-2}} .
\end{aligned}
$$

Similarly, we estimate, for every $s>1$,

$$
\begin{aligned}
\int_{A(k, R)} v_{h, k}^{\frac{p(p-1)}{p-2}} \eta^{\alpha p-p}|\nabla \eta|^{p} d x & \\
\leq & \left(\int_{A(k, R)} v_{h, k}^{\frac{p s(p-1)}{p-2}} d x\right)^{\frac{1}{s}}\left(\int_{A(k, R)} \eta^{(\alpha p-p) s^{\prime}}|\nabla \eta|^{p s^{\prime}} d x\right)^{\frac{1}{s^{\prime}}} .
\end{aligned}
$$


We deduce then from (4.13) that

$$
\begin{gathered}
\frac{1}{2}\left[\int_{\Omega}\left(v_{h, k}^{\frac{p-1}{p-2}} \eta^{\alpha}\right)^{p^{*}} d x\right]^{\frac{p}{p^{*}}} \leq C \int_{A(k, R)} \eta^{\alpha p} d x \\
+C \int_{A(k, R)}|\nabla \eta|^{\frac{2(p-1)}{p-2}} \eta^{\alpha p-\frac{2(p-1)}{p-2}} d x+C \int_{A(k, R)}|\nabla \eta|^{\frac{2 p}{p-2}} \eta^{\alpha p-\frac{2 p}{p-2}} d x \\
+\|f\|_{L^{q}\left(B_{R}\right)}^{\frac{p-1}{p-2}}\left(\int_{A(k, R)} \eta^{\alpha\left(p-p^{\prime}\right) \frac{q^{\prime} p^{*}}{p^{*}-q^{\prime} p^{\prime}}} d x\right)^{\left(\frac{1}{q^{\prime}}-\frac{p^{\prime}}{\left.p^{*}\right) \frac{p-1}{p-2}}\right.} \\
+\left\|v_{h, k}\right\|_{L^{\frac{p(p-1)}{p-2}}}^{\frac{s p(p-1)}{p-2}\left(B_{R}\right)}\left(\int_{A(k, R)} \eta^{\left.(\alpha p-p) s^{\prime}|\nabla \eta|^{p s^{\prime}} d x\right)^{\frac{1}{s^{\prime}}}}\right.
\end{gathered}
$$

We take $\alpha>\max \left(1, \frac{2}{p-2}\right)$, and in the right-hand side we use that $\eta \leq 1$ and $|\nabla \eta| \leq \frac{c}{R-\rho}$, and since $v_{h, k} \leq u^{+}$we obtain

$$
\begin{aligned}
& {\left[\int_{\Omega}\left(v_{h, k}^{\frac{p-1}{p-2}} \eta^{\alpha}\right)^{p^{*}} d x\right]^{\frac{p}{p^{*}}} \leq C|A(k, R)|\left(1+\frac{1}{(R-\rho)^{\frac{2(p-1)}{p-2}}}+\frac{1}{(R-\rho)^{\frac{2 p}{p-2}}}\right)} \\
& +\|f\|_{L^{q}\left(B_{R}\right)}^{\frac{p-1}{p-2}}|A(k, R)|^{\left(\frac{1}{q^{\prime}}-\frac{p^{\prime}}{p^{*}}\right) \frac{p-1}{p-2}}+\left\|u^{+}\right\|_{L^{\frac{p s(p-1)}{p-2}\left(B_{R}\right)}}^{\frac{p(p-1)}{(R-\rho)^{p}}}|A(k, R)|^{\frac{1}{s^{\prime}}} .
\end{aligned}
$$

Since $|A(k, R)|$ is bounded and $R-\rho \leq R$, we take $\mu=\max \left(p, \frac{2 p}{p-2}\right)$ and we choose suitably the value of $s>1$ in order to deduce (here is where we use the assumption $\left.q<N / p^{\prime}\right)$

$$
\left[\int_{\Omega}\left(v_{h, k}^{\frac{p-1}{p-2}} \eta^{\alpha}\right)^{p^{*}} d x\right]^{\frac{p}{p^{*}}} \leq K \frac{|A(k, R)|^{\left(\frac{1}{q^{\prime}}-\frac{p^{\prime}}{p^{*}}\right) \frac{p-1}{p-2}}}{(R-\rho)^{\mu}}
$$

where $K$ depends on $R,\|f\|_{L^{q}\left(B_{R}\right)}^{\frac{p-1}{p-2}},\left\|u^{+}\right\|_{L^{\frac{p s(p-1)}{p-2}}\left(B_{R}\right)}^{\frac{p(p-1)}{p-2}}$.

Recall that $\eta=1$ on $B_{\rho}$, hence we have, for any $h>k$,

$$
\begin{aligned}
\int_{\Omega}\left(v_{h, k}^{\frac{p-1}{p-2}} \eta^{\alpha}\right)^{p^{*}} d x \geq \int_{A(k, \rho)} v_{h, k}^{p^{*} \frac{p-1}{p-2}} & d x \\
& \geq \int_{A(h, \rho)} v_{h, k}^{p^{*} \frac{p-1}{p-2}} d x \geq(h-k)^{p^{*} \frac{p-1}{p-2}}|A(h, \rho)|
\end{aligned}
$$

and therefore we conclude

$$
|A(h, \rho)| \leq K^{\frac{p^{*}}{p}} \frac{|A(k, R)|^{\frac{p^{*}}{p}\left(\frac{1}{q^{\prime}}-\frac{p^{\prime}}{p^{*}}\right) \frac{p-1}{p-2}}}{(h-k)^{p^{*} \frac{p-1}{p-2}}(R-\rho)^{\mu \frac{p^{*}}{p}}} .
$$

One can check that

$$
\frac{p^{*}}{p}\left(\frac{1}{q^{\prime}}-\frac{p^{\prime}}{p^{*}}\right) \frac{p-1}{p-2}>1 \Longleftrightarrow q>\frac{N}{p}
$$


and we conclude applying the following lemma (see Lemma 5.1 in [15]):

Lemma 4.1. Let $\varphi(h, \rho):[0,+\infty) \times[0, R)$ be a function which is nonincreasing with respect to $h$ and nondecreasing with respect to $\rho$. Moreover, suppose that there exist $K_{0}>0, \tau>1$, and $C, \sigma, \delta>0$ such that

$$
\varphi(h, \rho) \leq \frac{C \varphi(k, R)^{\tau}}{(h-k)^{\sigma}(R-\rho)^{\delta}}, \quad \forall h>k>K_{0}, \forall \rho \in(0, R] .
$$

Then for every $s \in(0,1)$, there exists $d>0$ such that

$$
\varphi\left(K_{0}+d, s R\right)=0
$$

where

$$
d^{\sigma}=2^{\frac{\tau(\sigma+\delta)}{\tau-1}} C^{\prime} \frac{\varphi\left(K_{0}, 1\right)^{\tau-1}}{s^{\delta}} .
$$

Remark 4.4. If $q>\frac{N}{2}$, then similar local estimates are obtained in [12, even if for solutions rather than subsolutions. Note that $\frac{N}{p}<\frac{N}{2}$, so that the previous estimate is stronger and really exploits the superquadratic dependence of the nonlinearity.

Remark 4.5. Again we observe that the result of Theorem4.2 is still true if $a(x, s, \xi)$ verifies condition (3.6) with $g \in L_{\mathrm{loc}}^{\sigma}(\Omega), \sigma>\frac{N}{p-1}$.

Gathering together the above estimates with Lemma 3.1. we deduce universal estimates for positive subsolutions.

Corollary 4.1. Assume (2.2), let $2<p, \lambda>0$ and let $f$ belong to $L_{\mathrm{loc}}^{q}(\Omega)$ for some $q \geq 1$. Let $u \in W_{\text {loc }}^{1, p}(\Omega)$ be a subsolution of (2.1) in the sense of distributions. Then, for every pair of concentric balls $B_{\rho} \subset B_{R} \subset \Omega$, we have:

$$
\begin{array}{ll}
\text { if } q<\frac{N}{p}, & \left\|u^{+}\right\|_{L^{s}\left(B_{\rho}\right)} \leq K \quad \text { with } s=\frac{N p q}{N-p q}, \\
\text { if } q>\frac{N}{p}, & \left\|u^{+}\right\|_{L^{\infty}\left(B_{\rho}\right)} \leq K,
\end{array}
$$

where $K$ depends on $\beta, q, p, N, \lambda^{-1}, \rho, R,\|f\|_{L^{q}\left(B_{R}\right)}$ and on $\left\|\lambda u^{-}\right\|_{L^{1}\left(B_{R}\right)}$.

In particular, assume that $u \geq 0$; then (4.14) holds with a constant $K$ independent of $u$ and, moreover, if $q>\frac{N}{p}$ and $f \in L^{q}(\Omega)$, we have

$$
\|u\|_{L^{\infty}(\Omega)} \leq M
$$

where $M=M\left(\beta, q, p, N, \lambda^{-1}, \Omega,\|f\|_{L^{q}(\Omega)}\right)$.

Proof. The form of estimates (4.14) follows from Theorem 4.1 and Theorem 4.2 because of Lemma 3.1, which allows us to estimate $u^{+}$and $|\nabla u|^{p}$ in $L^{1}$ in terms of $u^{-}$.

The last statement is a consequence of Theorem 1.1. Indeed, a global bound on the oscillation of $u$ and a local $L^{\infty}$ bound, given by (4.14), imply the desired global estimate.

Similarly, the estimates are universal in the case of degenerate ellipticity.

Corollary 4.2. Assume (2.2), let $2<p, \lambda>0$ and let $f$ belong to $L_{\mathrm{loc}}^{q}(\Omega)$ for some $q \geq 1$. Let $u \in W_{\text {loc }}^{1, p}(\Omega)$ be a subsolution of (2.1) in the sense of distributions. Assume in addition that

$$
a(x, s, \xi) \cdot \xi \geq 0 \quad \forall(s, \xi) \in \mathbb{R} \times \mathbb{R}^{N} \text {, a.e. } x \in \Omega .
$$


Then estimates (4.14) hold true with a constant $K$ depending on $\beta, q, p, N, \lambda^{-1}$, $\rho, R$ and $\|f\|_{L^{q}\left(B_{R}\right)}$.

In addition, if $f \in L^{q}(\Omega)$ with $q>\frac{N}{p}$, then $u^{+} \in L^{\infty}(\Omega)$ and

$$
\left\|u^{+}\right\|_{L^{\infty}(\Omega)} \leq M
$$

where $M=M\left(\beta, q, p, N, \lambda^{-1}, \Omega,\|f\|_{L^{q}(\Omega)}\right)$.

Proof. Choosing $T_{\varepsilon}\left(u^{+}\right) \eta$ as the test function, where $\eta$ is a nonnegative cut-off function, we get

$$
\begin{aligned}
\lambda \int_{\Omega} u T_{\varepsilon}\left(u^{+}\right) \eta d x & +\int_{\Omega}|\nabla u|^{p} T_{\varepsilon}\left(u^{+}\right) \eta d x \leq \int_{\Omega} f T_{\varepsilon}\left(u^{+}\right) \eta d x \\
& -\int_{\Omega} a(x, u, \nabla u) \nabla T_{\varepsilon}\left(u^{+}\right) \eta d x-\int_{\Omega} a(x, u, \nabla u) \nabla \eta T_{\varepsilon}\left(u^{+}\right) d x
\end{aligned}
$$

Using (4.15) we can drop a term in the right-hand side, hence

$$
\begin{aligned}
\lambda \int_{\Omega} u T_{\varepsilon}\left(u^{+}\right) \eta d x+\int_{\Omega}|\nabla u|^{p} & T_{\varepsilon}\left(u^{+}\right) \eta d x \\
& \leq \int_{\Omega} f T_{\varepsilon}\left(u^{+}\right) \eta d x-\int_{\Omega} a(x, u, \nabla u) \nabla \eta T_{\varepsilon}\left(u^{+}\right) d x .
\end{aligned}
$$

Dividing by $\varepsilon$ and letting $\varepsilon \rightarrow 0$ we deduce that

$$
\lambda \int_{\Omega} u^{+} \eta d x+\int_{\Omega}\left|\nabla u^{+}\right|^{p} \eta d x \leq \int_{\Omega}|f| \eta d x-\int_{\Omega} a\left(x, u^{+}, \nabla u^{+}\right) \nabla \eta d x
$$

where we used also that $a(x, s, 0)=0$ as a consequence of (4.15). The above inequality means that $u^{+}$is a subsolution with right-hand side $|f|$. Then we apply Corollary 4.1 to conclude.

Remark 4.6. It is possible to give a more precise form of the dependence on the parameter $\lambda$ of the estimates in this section, by taking care of the scaling of the equation with respect to $\lambda$ (namely, applying the above arguments to the function $v=\lambda u$ ). In particular, one can replace estimate (4.1) with

$$
\min (\lambda, 1)\left\|u^{+}\right\|_{L^{s}\left(B_{\rho}\right)} \leq K
$$

and, respectively, estimate (4.9) with

$$
\min (\lambda, 1)\left\|u^{+}\right\|_{L^{\infty}\left(B_{\rho}\right)} \leq K
$$

where $K$ does not depend on $\lambda$. The same holds for the estimates in Corollary 4.1 and Corollary 4.2 ,

Remark 4.7. If (4.15) holds true and there exist $\gamma, L>0$ such that

$$
H(x, u, \nabla u) \operatorname{sign}(u) \geq \gamma|\nabla u|^{p} \quad \text { for }|u|>L,
$$

and if

$$
\lambda u-\operatorname{div}(a(x, u, \nabla u))+H(x, u, \nabla u)=f \quad \text { in } \Omega,
$$

then we get similar estimates for both $u^{+}$and $u^{-}$, proceeding as in Corollary 4.2. In particular, if $f \in L^{q}(\Omega)$ with $q>\frac{N}{p}$, we deduce a global universal bound $\|u\|_{L^{\infty}(\Omega)} \leq M$ (independent from the boundary behaviour of $u$ ). 


\section{Global Regularity for the Dirichlet problem}

We turn here to the Dirichlet problem, that is, we assume that the subsolution $u$ belongs to the space $W_{0}^{1, p}(\Omega)$. In this case, we find global summability or regularity, depending on the summability of the datum $f$. We stress the fact that, in the next two results, $\lambda$ can be any real number.

\subsection{Global $L^{s}$-regularity.}

Theorem 5.1. Assume (2.2), let $2<p<N, \lambda \in \mathbb{R}$ and let $f$ belong to $L^{q}(\Omega)$ for some $q$ such that

$$
1 \leq q<\frac{N}{p} .
$$

Let $u \in W_{0}^{1, p}(\Omega)$ be a subsolution of (2.1) in the sense of distributions. Then

$$
u \in L^{s}(\Omega), \quad \text { with } s=\frac{N p q}{N-p q},
$$

and

$$
\|u\|_{L^{s}(\Omega)} \leq C
$$

where the bound $C$ depends on $\beta, p, N, q, \lambda,|\Omega|,\|f\|_{L^{q}(\Omega)}$ in the case where $\frac{N(p-1)}{N(p-2)+p}$ $\leq q<\frac{N}{p}$, while it also depends on the $L^{p}$-norm of $|\nabla u|$ in the case where $1 \leq q<$ $\frac{N(p-1)}{N(p-2)+p}$.

Proof. It is easy to see that (2.3) must be true for every $\varphi \in L^{\infty}(\Omega) \cap H_{0}^{1}(\Omega)$. Let us start by assuming that

$$
\frac{N(p-1)}{N(p-2)+p} \leq q<\frac{N}{p}
$$

Take $\varphi=\left|T_{k}(u)\right|^{\frac{N p(q-1)}{N-p q}}$ and use (2.2) to obtain

$$
\begin{aligned}
\int_{\Omega}|\nabla u|^{p}\left|T_{k}(u)\right|^{\frac{N p(q-1)}{N-p q}} d x \leq C \int_{\Omega}\left|\nabla T_{k}(u)\right|^{2}\left|T_{k}(u)\right|^{\frac{N p(q-1)}{N-p q}-1} d x \\
+C \int_{\Omega}\left|\nabla T_{k} u\right|\left|T_{k}(u)\right|^{\frac{N p(q-1)}{N-p q}-1} d x \\
+|\lambda| \int_{\Omega}|u|\left|T_{k}(u)\right|^{\frac{N p(q-1)}{N-p q}} d x+\int_{\Omega}|f|\left|T_{k}(u)\right|^{\frac{N p(q-1)}{N-p q}} d x .
\end{aligned}
$$

Here the constants $C$ depend on the data of the problem but not on $k$ (and may change from line to line). We now proceed to estimate the integrals in (5.4). If we set

$$
\Phi_{k}(t)=\int_{0}^{|t|}\left(T_{k}(s)\right)^{\frac{N(q-1)}{N-p q}} d s,
$$

then, using Sobolev's inequality, one obtains

$$
\begin{aligned}
\int_{\Omega}|\nabla u|^{p}\left|T_{k}(u)\right|^{\frac{N p(q-1)}{N-p q}} d x=\int_{\Omega}\left|\nabla \Phi_{k}(u)\right|^{p} d x \\
\geq C\left[\int_{\Omega} \Phi_{k}(u)^{p^{*}} d x\right]^{\frac{N-p}{N}} \geq C\left[\int_{\Omega}\left|T_{k}(u)\right|^{s} d x\right]^{\frac{N-p}{N}} .
\end{aligned}
$$


On the other hand, by Young's and Hölder's inequalities, we can write

$$
\begin{aligned}
\int_{\Omega} \mid & \left.\nabla T_{k}(u)\right|^{2}\left|T_{k}(u)\right|^{\frac{N p(q-1)}{N-p q}-1} d x \\
& \leq \varepsilon \int_{\Omega}|\nabla u|^{p}\left|T_{k}(u)\right|^{\frac{N p(q-1)}{N-p q}} d x+C_{\varepsilon} \int_{\Omega}\left|T_{k}(u)\right|^{\frac{N p(q-1)}{N-p q}-\frac{p}{p-2}} d x \\
& \leq \varepsilon \int_{\Omega}|\nabla u|^{p}\left|T_{k}(u)\right|^{\frac{N p(q-1)}{N-p q}} d x+C_{\varepsilon}\left[\int_{\Omega}\left|T_{k}(u)\right|^{s} d x\right]^{\frac{q-1}{q}-\frac{N-p q}{N q(p-2)}}
\end{aligned}
$$

for arbitrary $\varepsilon>0$ (note that the lower bound on $q$ in (5.3) ensures that $\frac{N p(q-1)}{N-p q}-$ $\left.\frac{p}{p-2} \geq 0\right)$. Similarly

$$
\begin{aligned}
& \int_{\Omega}\left|\nabla T_{k}(u)\right|\left|T_{k}(u)\right|^{\frac{N p(q-1)}{N-p q}-1} d x \\
& \leq \varepsilon \int_{\Omega}|\nabla u|^{p}\left|T_{k}(u)\right|^{\frac{N p(q-1)}{N-p q}} d x+C_{\varepsilon} \int_{\Omega}\left|T_{k}(u)\right|^{\frac{N p(q-1)}{N-p q}-\frac{p}{p-1}} d x \\
& \leq \varepsilon \int_{\Omega}|\nabla u|^{p}\left|T_{k}(u)\right|^{\frac{N p(q-1)}{N-p q}} d x+C_{\varepsilon}\left[\int_{\Omega}\left|T_{k}(u)\right|^{s} d x\right]^{\frac{q-1}{q}-\frac{N-p q}{N q(p-1)}} .
\end{aligned}
$$

Moreover, by Hölder's inequality,

$$
\int_{\Omega}|u|\left|T_{k}(u)\right|^{\frac{N p(q-1)}{N-p q}} d x \leq C\left[\int_{\Omega}\left(|u|\left|T_{k}(u)\right|^{\frac{N p(q-1)}{N-p q}}\right)^{\frac{s}{1+\frac{N p(q-1)}{N-p q}}}\right]^{\left(1+\frac{N p(q-1)}{N-p q}\right) \frac{1}{s}} .
$$

Now, one can easily check that

$$
\left(|u|\left|T_{k}(u)\right|^{\frac{N p(q-1)}{N-p q}}\right)^{\frac{s}{1+\frac{N p(q-1)}{N-p q}}} \leq C \Phi_{k}(u)^{p^{*}}
$$

therefore,

$$
\int_{\Omega}|u|\left|T_{k}(u)\right|^{\frac{N p(q-1)}{N-p q}} d x \leq C\left[\int_{\Omega} \Phi_{k}(u)^{p^{*}}\right]^{\left(1+\frac{N p(q-1)}{N-p q}\right) \frac{1}{s}} .
$$

Finally

$$
\int_{\Omega}|f|\left|T_{k}(u)\right|^{\frac{N p(q-1)}{N-p q}} d x \leq\|f\|_{L^{q}(\Omega)}\left[\int_{\Omega}\left|T_{k}(u)\right|^{s} d x\right]^{\frac{1}{q^{\prime}}} .
$$

Therefore, putting all the inequalities (5.4)-(5.9) together, we obtain

$$
\begin{aligned}
X \leq c_{10}\left(X^{\left(\frac{q-1}{q}-\frac{N-p q}{N q(p-2)}\right) \frac{N}{N-p}}+X^{\left(\frac{q-1}{q}-\frac{N-p q}{N q(p-1)}\right) \frac{N}{N-p}}\right. & \\
& \left.+X^{\frac{N-p q+N p(q-1)}{p q(N-p)}}+X^{\frac{N(q-1)}{(N-p) q}}\right),
\end{aligned}
$$

where $c_{10}$ depends on $\beta, N, p, q, \lambda,|\Omega|,\|f\|_{L^{q}(\Omega)}$, and we have set

$$
X=\int_{\Omega}|\nabla u|^{p}\left|T_{k}(u)\right|^{\frac{N p(q-1)}{N-p q}} d x .
$$

Since $q<N / p$, it is easy to check that all the four exponents in the right-hand side of (5.10) are smaller than 1 . This gives an estimate on $X$, therefore, on $\int_{\Omega}\left|T_{k}(u)\right|^{s} d x$. The result follows by letting $k$ go to infinity. 
In the case where

$$
1 \leq q<\frac{N(p-1)}{N(p-2)+p}
$$

the above proof does not work. However, using

$$
\left(1+\left|T_{k}(u)\right|\right)^{\frac{N p(q-1)}{N-p q}}-1
$$

as the test function, with the same type of calculations as in the proof of Theorem 4.1, it is easy to prove the same result, the only difference being that in this case the bound $C$ in (5.2) also depends on the $L^{p}$-norm of $|\nabla u|$.

5.2. Global boundedness. We now need the following lemma (see [14]):

Lemma 5.1. Let $\phi$ be a nonnegative, nonincreasing function defined on the half line $\left[k_{0}, \infty\right)$. Suppose that there exist positive constants $A, \gamma, \delta$, with $\delta>1$, such that

$$
\phi(h) \leq \frac{A}{(h-k)^{\gamma}} \phi(k)^{\delta}
$$

for every $h>k \geq k_{0}$. Then $\phi(k)=0$ for every $k \geq k_{1}$, where

$$
k_{1}=k_{0}+A^{1 / \gamma} 2^{\delta /(\delta-1)} \phi\left(k_{0}\right)^{(\delta-1) / \gamma} .
$$

Theorem 5.2. Assume (2.2), let $p>2, \lambda \in \mathbb{R}$, and let $f$ belong to $L^{q}(\Omega)$ for some $q>\frac{N}{p}$. Let $u \in W_{0}^{1, p}(\Omega)$ be a subsolution of (2.1) in the sense of distributions. Then $u \in L^{\infty}(\Omega)$, and

$$
\|u\|_{L^{\infty}(\Omega)} \leq C\left(\beta, p, N, q, \lambda,\|f\|_{L^{q}(\Omega)},|\Omega|\right) .
$$

Proof. By using the usual inclusions between Lebesgue spaces, one can always suppose that

$$
\frac{N}{p}<q \leq \frac{N}{p^{\prime}}
$$

Moreover, by Theorem [5.1, $u \in L^{s}(\Omega)$ for every $s>1$ (with norm depending on the data of the problem). Therefore, we can put the term $\lambda u$ in the equation with the datum $f$, and we can ignore it. It is easy to see that (2.3) is true for every $\varphi \in L^{\infty}(\Omega) \cap H_{0}^{1}(\Omega)$. We take $\varphi=\left(G_{k, h}(u)\right)^{\frac{p}{p-2}}$ in (2.3), where $h>k>0$

$$
G_{h, k}(s)=T_{h-k}(|s|-k)_{+} .
$$

Then we obtain

$$
\begin{aligned}
& \int_{A_{k}}|\nabla u|^{p}\left(G_{k, h}(u)\right)^{\frac{p}{p-2}} d x \\
& \quad \leq \beta \frac{p}{p-2} \int_{A_{k}}\left(|\nabla u|^{2}+|\nabla u|\right)\left(G_{k, h}(u)\right)^{\frac{2}{p-2}} d x+\int_{A_{k}}|f|\left(G_{k, h}(u)\right)^{\frac{p}{p-2}} d x,
\end{aligned}
$$

where we have set

$$
A_{k}=\{x \in \Omega:|u(x)|>k\} .
$$

Then, using Young' inequality, we obtain

$$
\begin{aligned}
& \int_{A_{k}} \mid\left.\nabla u\right|^{p}\left(G_{k, h}(u)\right)^{\frac{p}{p-2}} d x \\
& \quad \leq \frac{1}{2} \int_{A_{k}}|\nabla u|^{p}\left(G_{k, h}(u)\right)^{\frac{p}{p-2}} d x+c_{1}\left|A_{k}\right|+\int_{A_{k}}\left(|f|+c_{1}\right)\left(G_{k, h}(u)\right)^{\frac{p}{p-2}} d x,
\end{aligned}
$$


where $c_{1}=c_{1}(\beta, p)$. Therefore, if we set $\tilde{f}=|f|+c_{1}$, we obtain

$$
\int_{A_{k}}|\nabla u|^{p}\left(G_{k, h}(u)\right)^{\frac{p}{p-2}} d x \leq C\left|A_{k}\right|+2 \int_{A_{k}} \tilde{f}\left(G_{k, h}(u)\right)^{\frac{p}{p-2}} d x .
$$

On the other hand, by Sobolev's inequality,

$$
\begin{aligned}
\int_{A_{k}}|\nabla u|^{p}\left(G_{k, h}(u)\right)^{\frac{p}{p-2}} d x & =C \int_{A_{k}}\left|\nabla\left(G_{k, h}(u)\right)^{\frac{p-1}{p-2}}\right|^{p} d x \\
& \geq C\left[\int_{A_{k}}\left(G_{k, h}(u)\right)^{\frac{(p-1) p^{*}}{p-2}} d x\right]^{\frac{p}{p^{*}}},
\end{aligned}
$$

while

$$
\begin{array}{r}
\int_{A_{k}} \tilde{f}\left(G_{k, h}(u)\right)^{\frac{p}{p-2}} d x \leq\|\tilde{f}\|_{L^{q}(\Omega)}\left[\int_{A_{k}}\left(G_{k, h}(u)\right)^{\frac{(p-1) p^{*}}{p-2}} d x\right]^{\frac{p^{\prime}}{p^{*}}}\left|A_{k}\right|^{1-\frac{1}{q}-\frac{p^{\prime}}{p^{*}}} \\
\leq \varepsilon\left[\int_{A_{k}}\left(G_{k, h}(u)\right)^{\frac{(p-1) p^{*}}{p-2}} d x\right]^{\frac{p}{p^{*}}}+C_{\varepsilon}\|\tilde{f}\|_{L^{q}(\Omega)}^{\frac{p-1}{p-2}}\left|A_{k}\right|^{\left(\frac{1}{q^{\prime}}-\frac{p^{\prime}}{p^{*}}\right) \frac{p-1}{p-2}} .
\end{array}
$$

Therefore, we have found that

$$
\begin{aligned}
{\left[\int_{A_{k}}\left(G_{k, h}(u)\right)^{\frac{(p-1) p^{*}}{p-2}} d x\right]^{\frac{p}{p^{*}}} } & \leq C\left(\left|A_{k}\right|+\left|A_{k}\right|^{\left(\frac{1}{q^{\prime}}-\frac{p^{\prime}}{p^{*}}\right) \frac{p-1}{p-2}}\right) \\
& \leq C\left|A_{k}\right|^{r}
\end{aligned}
$$

where

$$
r=\left(\frac{1}{q^{\prime}}-\frac{p^{\prime}}{p^{*}}\right) \frac{p-1}{p-2},
$$

while $C$ depends on $\beta, p, N,\|f\|_{L^{q}(\Omega)}$ and $|\Omega|$. In the last inequality we have used that $1 \geq r$, which follows from the assumption (5.12). Since $G_{k, h}(u)=h-k$ on $A_{h}$, one obtains

$$
(h-k)^{\frac{(p-1) p}{p-2}}\left|A_{h}\right|^{\frac{p}{p^{*}}} \leq C\left|A_{k}\right|^{r},
$$

that is,

$$
\left|A_{h}\right| \leq \frac{C^{\frac{p^{*}}{p}}}{(h-k)^{\frac{(p-1) p^{*}}{p-2}}}\left|A_{k}\right|^{\frac{p^{*} r}{p}} .
$$

It is easy to check that $\frac{p^{*} r}{p}>1$, therefore, we can apply Lemma 5.1 to $\phi(k)=\left|A_{k}\right|$. Note that, by the results of the previous section, $u$ is estimated in $L^{\sigma}(\Omega)$ for every $\sigma<\infty$, therefore, for a fixed $k_{0}>0\left|A_{k_{0}}\right|$ can be estimated in terms of the data.

Remark 5.1. Note that this result is false if $p \leq 2$. Indeed, one may find unbounded distributional solutions even when $f \equiv 0$. See [1] for the case $p=2$, or [10] for the subquadratic case and a discussion of the bootstrap property for weak solutions.

We also refer to Remark 3.2 for a simple example showing the optimality of Hölder regularity for distributional solutions and, at the same time, that this regularity is not enough to yield uniqueness of distributional solutions of the Dirichlet problem. 


\section{References}

[1] Boumediene Abdellaoui, Andrea Dall'Aglio, and Ireneo Peral, Some remarks on elliptic problems with critical growth in the gradient, J. Differential Equations 222 (2006), no. 1, 21-62, DOI 10.1016/j.jde.2005.02.009. MR2200746 (2006k:35070)

[2] Guy Barles, A short proof of the $C^{\overline{0}, \alpha}$-regularity of viscosity subsolutions for superquadratic viscous Hamilton-Jacobi equations and applications, Nonlinear Anal. 73 (2010), no. 1, 31-47, DOI 10.1016/j.na.2010.02.009. MR2645829(2011f:35047)

[3] L. Boccardo, F. Murat, and J.-P. Puel, $L^{\infty}$ estimate for some nonlinear elliptic partial differential equations and application to an existence result (English, with French summary), SIAM J. Math. Anal. 23 (1992), no. 2, 326-333, DOI 10.1137/0523016. MR1147866 (93d:35049)

[4] Piermarco Cannarsa and Pierre Cardaliaguet, Hölder estimates in space-time for viscosity solutions of Hamilton-Jacobi equations, Comm. Pure Appl. Math. 63 (2010), no. 5, 590-629, DOI 10.1002/cpa.20315. MR2583307 (2012c:35203)

[5] Pierre Cardaliaguet, A note on the regularity of solutions of Hamilton-Jacobi equations with superlinear growth in the gradient variable, ESAIM Control Optim. Calc. Var. 15 (2009), no. 2, 367-376, DOI 10.1051/cocv:2008028. MR2513090(2010e:35047)

[6] Pierre Cardaliaguet and Luis Silvestre, Hölder continuity to Hamilton-Jacobi equations with superquadratic growth in the gradient and unbounded right-hand side, Comm. Partial Differential Equations 37 (2012), no. 9, 1668-1688, DOI 10.1080/03605302.2012.660267. MR.2969493

[7] Pierre Cardaliaguet and Catherine Rainer, Hölder regularity for viscosity solutions of fully nonlinear, local or nonlocal, Hamilton-Jacobi equations with superquadratic growth in the gradient, SIAM J. Control Optim. 49 (2011), no. 2, 555-573, DOI 10.1137/100784400. MR2784700(2012d:49053)

[8] I. Capuzzo Dolcetta, F. Leoni, and A. Porretta, Hölder estimates for degenerate elliptic equations with coercive Hamiltonians, Trans. Amer. Math. Soc. 362 (2010), no. 9, 45114536, DOI 10.1090/S0002-9947-10-04807-5. MR2645039 (2011c:35178)

[9] David Gilbarg and Neil S. Trudinger, Elliptic partial differential equations of second order, 2nd ed., Grundlehren der Mathematischen Wissenschaften [Fundamental Principles of Mathematical Sciences], vol. 224, Springer-Verlag, Berlin, 1983. MR737190 (86c:35035)

[10] Nathalie Grenon, François Murat, and Alessio Porretta, A priori estimates and existence for elliptic equations with gradient dependent terms, Ann. Sc. Norm. Super. Pisa Cl. Sci. (5) 13 (2014), no. 1, 137-205. MR 3235059

[11] J.-M. Lasry and P.-L. Lions, Nonlinear elliptic equations with singular boundary conditions and stochastic control with state constraints. I. The model problem, Math. Ann. 283 (1989), no. 4, 583-630, DOI 10.1007/BF01442856. MR990591 (90f:35072)

[12] Tommaso Leonori, Large solutions for a class of nonlinear elliptic equations with gradient terms, Adv. Nonlinear Stud. 7 (2007), no. 2, 237-269. MR2308039 (2008c:35080)

[13] P.-L. Lions, Quelques remarques sur les problèmes elliptiques quasilinéaires du second ordre (French), J. Analyse Math. 45 (1985), 234-254, DOI 10.1007/BF02792551. MR833413 (87f:35088)

[14] Guido Stampacchia, Èquations elliptiques du second ordre à coefficients discontinus (French), Séminaire de Mathématiques Supérieures, No. 16 (Été, 1965), Les Presses de l'Université de Montréal, Montreal, Que., 1966. MR0251373 (40 \#4603)

[15] Guido Stampacchia, Le problème de Dirichlet pour les équations elliptiques du second ordre à coefficients discontinus (French), Ann. Inst. Fourier (Grenoble) 15 (1965), no. fasc. 1, 189-258. MR0192177 (33 \#404)

Dipartimento di Matematica, Università di Roma "La Sapienza", Piazzale Aldo Moro 5 - 00185 Roma, ITALY

E-mail address: dallaglio@mat.uniroma1.it

Dipartimento di Matematica, Università di Roma Tor Vergata, Via della Ricerca SCIENTIFICA - 00133 Roma, Italy

E-mail address: porretta@mat.uniroma2.it 